Peer Reviewed Paper openaccess

\title{
Restoration of defaced serial numbers using lock-in infrared thermography (Part II)
}

\author{
Ikwulono Unobe, ${ }^{a}$ Lisa Lau, ${ }^{\mathrm{b}}$ John Kalivas, ${ }^{\mathrm{c}, *}$ Rene Rodriguez, ${ }^{\mathrm{d}, *}$ and Andrew Sorensen ${ }^{\mathrm{e}}$ \\ ${ }^{a}$ College of Science and Engineering, Idaho State University, 921 S. $8^{\text {th }}$ Avenue, Stop 8023, Pocatello, ID, 83209-8023, USA. \\ E-mail: unobikwu@isu.edu, https://orcid.org/0000-0001-7145-5027 \\ ${ }^{b}$ College of Science and Engineering, Idaho State University, 921 S. $8^{\text {th }}$ Avenue, Stop 8023, Pocatello, ID, 83209-8023, USA. \\ E-mail: laulisa@isu.edu, https://orcid.org/0000-0001-8872-3390 \\ ${ }^{c}$ College of Science and Engineering, Idaho State University, 921 S. $8^{\text {th }}$ Avenue, Stop 8023, Pocatello, ID, 83209-8023, USA. \\ E-mail: kalijohn@isu.edu, https://orcid.org/0000-0001-7056-976X \\ ${ }^{d}$ College of Science and Engineering, Idaho State University, 921 S. $8^{\text {th }}$ Avenue, Stop 8023, Pocatello, ID, 83209-8023, USA. \\ E-mail: rodrrene@isu.edu, https://orcid.org/0000-0003-4878-0299 \\ eDepartment of Civil and Environmental Engineering, Utah State University, 4110 Old Main Hill, Logan, Utah, 84322-4110, USA. \\ E-mail: andrew.sorensen@usu.edu, https://orcid.org/0000-0001-9998-2021
}

This paper details continuing work on the development of a substantive non-destructive method to recover defaced serial numbers stamped or laser engraved into metallic objects based on lock-in infrared thermography. This method relies on the existence of a local zone of plastic strain created from stamping pressures in mechanically stamped pieces and a heat-affected zone in laser engraved samples, both extending to depths below the visible characters. The grain structure within these zones is dislocated due to the external forces applied. These deformed areas are exposed to the surface when the serial numbers are defaced. Infrared thermography utilises the change in thermal conductivity, based on local variation in the thermal gradient from thermal energy applied at the surface. Observation of the thermal gradient at the surface as it propagates through the object allows for identification of these deformed regions and subsequent recovery of the serial number. Principal component analysis is used to enhance the thermographic images. Pseudo Zernike moments and subsequently multiple similarity measures are utilised to identify the numbers based on a reference library of numbers. Several fusion rules are used to obtain a consensus across the similarity measures. This process is tested on several datasets including stamped numbers on a gun barrel, laser engraved numbers on a needle holder and the defaced vehicle identification number of a stolen motorcycle.

Keywords: serial number restoration, lock-in infrared thermography, principal component analysis, pseudo Zernike moments, similarity measure, fusion

\section{Introduction}

Serial numbers are usually stamped using a die designed to imprint characters by applying sufficient pressure to impart a permanent impression of the die symbol into an object. This stamping pressure causes a permanent
Correspondence

John Kalivas (kalijohn@isu.edu) and Rene Rodriguez (rodrrene@isu.edu)

Received: 8 April 2019

Revised: 20 October 2019

Accepted: 1 November 2019

Publication: 18 November 2019

doi: 10.1255/jsi.2019.a20

ISSN: $2040-4565$

\begin{abstract}
Citation
I. Unobe, L. Lau, J. Kalivas, R. Rodriguez and A. Sorensen, "Restoration of defaced serial numbers using lock-in infrared thermography (Part II)", J.Spectral Imaging 8, a19 (2019). https://doi.org/10.1255/jsi.2019.a20

(C) 2019 The Authors

This licence permits you to use, share, copy and redistribute the paper in any medium or any format provided that a full citation to the original paper in this journal is given, the use is not for commercial purposes and the paper is not changed in any way.
\end{abstract}


change in geometry (plastic deformation) leading to an alteration of the local crystalline structure and the interlocking grain boundaries. The resulting deformation extends to some depth below the visible stamped mark and is known as the zone of plastic strain. This plastic strain zone possesses physical and chemical properties differing from those of the original un-stamped metal $^{1}$ with local changes to properties such as hardness, magnetisation behaviour, thermal conductivity and chemical potential. Each of these property changes forms the basis for different serial number recovery methods. $^{2}$

A more recent method of applying serial numbers increasingly used in firearms and automobile marking is laser engraving. The laser engraving process marks the metal using the intense heat generated by a focused laser beam to alter the structure in the material. This process also liquefies the region below the engraved number and the rapid cooling of the metal may not allow for the reformation of the deformed crystals into the original arrangement creating what is referred to as a heat-affected zone (HAZ). This process, unlike mechanical stamping, leaves no cold worked or plastic strain region. Instead, the altered material structure within the HAZ leads to changes in properties similar to that for the plastic strain region. ${ }^{3,4}$

Some well-documented methods for restoring defaced serial numbers include chemical etching, heat treatment, ultrasonic cavitation and electrolytic etching., ${ }^{3,5}$ All of these techniques find a commonality in being nonrepeatable due to their destructive nature. Also, the lack of change in the reduction potential of the HAZ reduces the effectiveness of the well-established chemical etching recovery process for laser engraved serial numbers. Magnetic particle inspection is a non-destructive restoration technique that leverages differences in magnetic permeability within the zone of plastic strain to recover the defaced serial numbers ${ }^{6}$ but is only applicable to ferromagnetic alloys and magnetic metals.

A non-destructive alternative is infrared thermography. This method was recently developed and evaluated using numbers stamped in a steel plate. ${ }^{7}$ The process involves applying thermal energy to the surface of an object. As the thermal energy propagates through the object, infrared images are collected over the time of propagation to capture thermal gradient differences across the surface. The thermal gradient differences occur in regions of plastic strain or within the HAZ due to the local change in thermal conductivity. These differences allow restoration of the defaced number.

The process used previously ${ }^{7}$ requires inducing a temperature change about some starting temperature and collecting thermal images for a particular amount of time to cover the period of the pulsing heat frequency used to induce the temperature change. These images are then used to develop amplitude and phase images using lock-in thermography (LIT) principles. The developed amplitude and phase images are further processed using principal component analysis (PCA) to characterise and enhance the thermal gradient differences in the deformed areas within a score image or subset of score images.

Subsequently, after utilising PCA to develop score images that characterise these defaced regions, it is pertinent to decisively identify the numbers delineated by the local thermal gradient changes within the score images. This identification is carried out by comparing the score images to the digits in a reference number library using similarity measures and high-level data fusion $n^{7,8}$ to obtain a match for the score images to a number.

Identifying the defaced numbers from score images using similarity measures requires the extraction of suitable image features to be used in comparing the images to those of a reference library. In order to accurately match the images, the extracted features need to be robust to possible size, scale, translation or rotation dissimilarities between the score images and those of the reference library. Orthogonal moments are a proven way of extracting features invariant to these differences. One such orthogonal moment, proven to be quite robust for image classification is the pseudo Zernike moment (PZM). The PZMs are used to extract rotationally invariant features from an image and have been shown to outperform other orthogonal moments including regular Zernike moments in the presence of noise. ${ }^{9-11}$ Preprocessing the images using regular moments to make them scale and translation invariant ensures that the extracted PZM features are scale, translation and rotation invariant.

Previous work employing this laid out procedure showed some success in recovering and identifying defaced serial numbers from a simulated stainless steel test piece. ${ }^{7}$ This study further tests the process using improvements noted in the Number identification section. Samples evaluated are a gun barrel, needle holder with laser engraved markings and the vehicle identification number (VIN) of a recovered stolen motorcycle. 


\section{Materials and methodology}

\section{Test specimens}

Gun barrel

The barrel of a Stevens Model 9512 gauge shotgun was acquired for use as a test piece. The shotgun had no serial number and hence, two numbers stamped on the barrel were defaced using a file. Figures $1 \mathrm{a}$ and $1 \mathrm{~b}$ show images of the barrel before and after defacing.

Needle holder

To test the efficacy of the method on laser engraved samples, a laser engraved Aesculap surgical needle holder (Model BM034R) was obtained. Two of the laser engraved numbers were filed to simulate defacing serial numbers. Figures $2 \mathrm{a}$ and $2 \mathrm{~b}$ shows the needle holder before and after the characters are removed.

\section{Motorcycle VIN}

A recovered Yamaha model TY175 motorcycle with the VIN defaced on the fork ostensibly to prevent identification was obtained from the Idaho Power County Sheriff's Department for use to test the process. The motorcycle's defaced VIN on the fork is presented in Figure 3. This specimen had the VIN completely removed (Figure 3a)
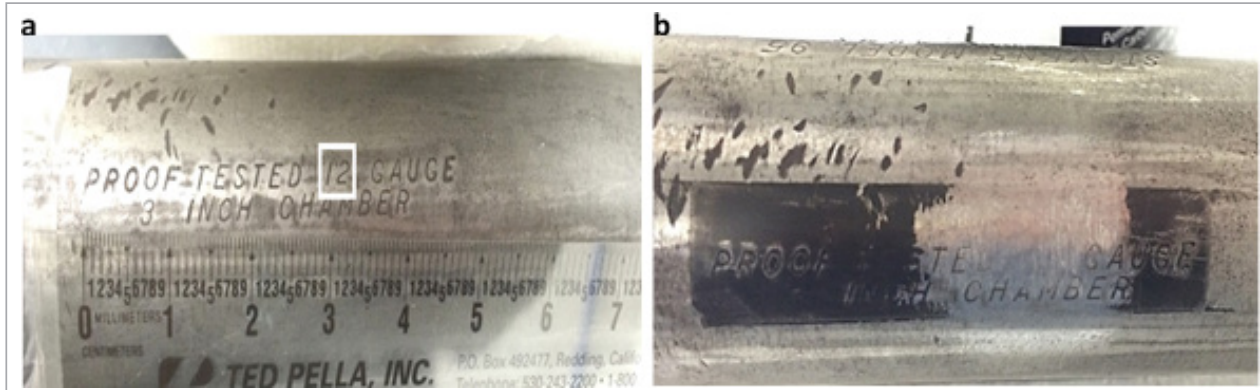

Figure 1. Gun barrel with a) identification marks stamped in and b) numbers defaced. (The India ink coating has been partially removed from the areas with the numbers 1 and 2 for ease of viewing.)

a

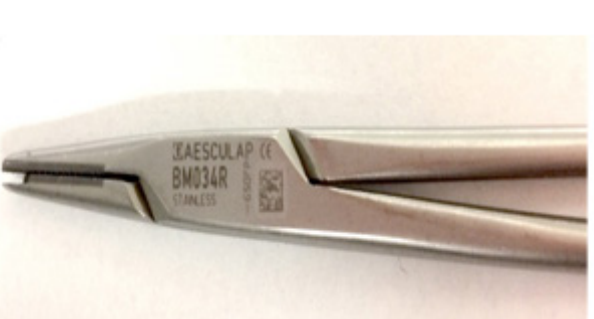

Figure 2. Aesculap needle holder with a) laser engraved numbers and b) numbers defaced and painted with India ink.
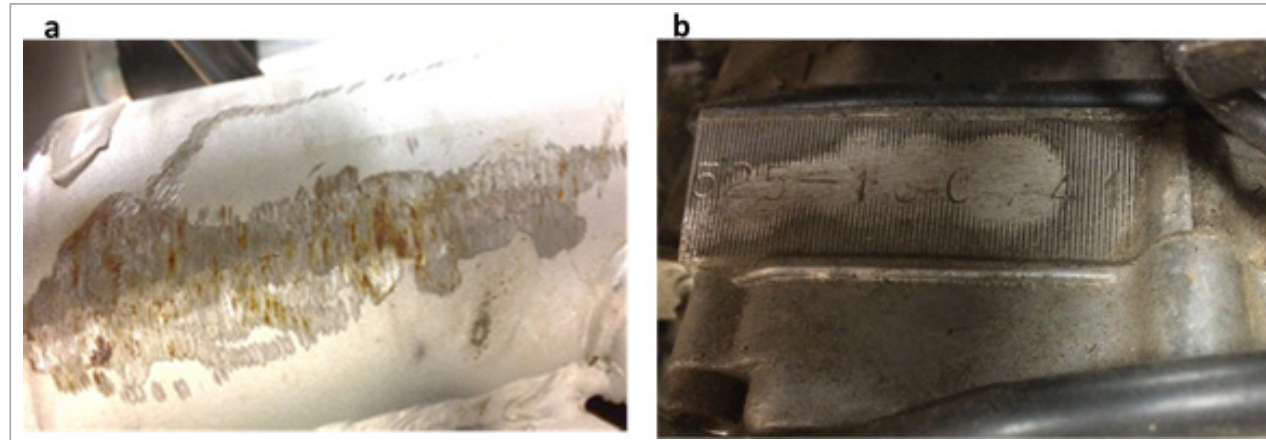

Figure 3. a) Defaced VIN on motorcycle fork and b) VIN on motorcycle engine with most numbers still intact.

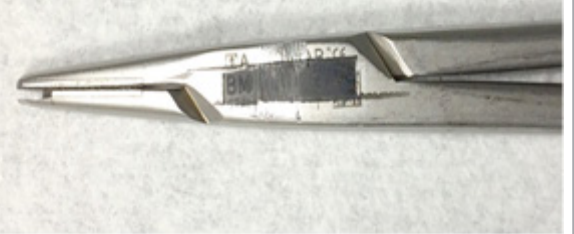


but the VIN stamped into the engine block (Figure 3b) only had a few of the numbers defaced and was used to confirm the numbers delineated in the score images of the fork. This verification process assumes the engine block and fork are original equipment and thus, the numbers on them will be the same.

\section{Sample preparation}

The surface areas on all the acquired samples are polished using a 600 grit sandpaper to ensure a smooth surface, carefully cleaned with ethanol and covered with a light coat of India ink $(\sim 30 \mathrm{~nm})$ to reduce the possible adverse effects from surface emissivity on the quality of the captured images.

\section{Data collection and image enhancement}

The experimental system, as described previously, ${ }^{7}$ consists of an infrared camera, a function generator and thermal energy source. A digital hot plate is used to heat up some of the samples (steel plate, gun barrel and needle holder) to an initial temperature of approximately $73^{\circ} \mathrm{C}$ as measured by a digital thermometer. This is done to ensure that the samples are at an even initial temperature and to enhance the contrast of the thermal gradient across the surface as recommended previously ${ }^{12}$ to improve contrast. However, due to size and shape constraints, it is not possible to use this setup for the motorcycle fork. Therefore, heating tape is wrapped around the fork in lieu of the hot plate and used to heat the fork up to an initial temperature of approximately $70^{\circ} \mathrm{C}$ which, although not entirely even over the area examined, proved adequate for the experiments.

The data collection and image enhancement protocol used in this study is a two-step process as described in previous work. ${ }^{7}$ The first step utilises LIT to collect infrared thermal images over several pulsing cycles and develop phase and amplitude images for each cycle. The temperature distribution on the heated surface is monitored using the infrared camera to collect images at a frame rate of 32 frames per laser pulse cycle over several cycles to continuously capture the irregular heat propagation through the zone of plastic strain or HAZ created in the process of applying the number. The irregular propagation becomes more evident with the inclusion of more pulses as any noise in the thermal images associated propagation of the thermal energy is averaged out with the inclusion of more pulses. During the experiments, thermal energy is pulsed into the surface of the sample under observation using the laser at a defined modulated (lock-in) frequency. The initial experiments in employing this process reported previously ${ }^{7}$ used a lock-in frequency of $0.05 \mathrm{~Hz}$ to modulate the thermal energy input into the sample. The results from that study indicate that it is a satisfactory modulation frequency for this process. However, the phase difference plot developed in the study ${ }^{7}$ also indicated that other modulation frequencies could prove equally as good in capturing the defects within the zone of plastic strain and reproduce the defaced number. As such, testing was extended to four modulation frequencies for each sample to determine their effectiveness in capturing the change in thermal gradient necessary for recovering the defaced numbers. These frequencies are $1 \mathrm{~Hz}, 0.125 \mathrm{~Hz}, 0.05 \mathrm{~Hz}$ and $0.03125 \mathrm{~Hz}$ corresponding to $1 \mathrm{~s}, 8 \mathrm{~s}, 20 \mathrm{~s}$ and $32 \mathrm{~s}$ pulse cycle times, respectively. These frequencies are selected from the phase difference plot described in previous work ${ }^{7}$ for the defaced and non-defaced areas of a stainless steel plate.

Testing across several modulation frequencies leverages on a characteristic of LIT which is that the depth of penetration for flaw characterisation in a material is inversely proportional to the modulation frequency. ${ }^{13}$ Thus, while shallower defects can be visualised in the phase images from higher frequencies, deeper flaws require lower modulation frequencies to be captured. Utilising the four modulation frequencies allows for investigating a range of depths within the material for the depth at which the defaced number is best characterised; as this could vary with respect to the method of applying the serial numbers, the material and the degree of defacing.

Thermographic images can be noisy due to undesired signals from several factors including unevenly heated surfaces, radiation from the heated surface and local emissivity variations. Developing phase and amplitude images helps to mitigate some of these effects. Although the phase image is usually more robust to these effects, the amplitude images does in some cases provide images with enough contrast to identify the thermal gradients present in areas of local plastic strain. Development of the amplitude and phase images is accomplished by a digital lock-in correlation procedure as described in the literature ${ }^{14}$ with some modifications as explained previously. ${ }^{7}$

Each pulse cycle is used to develop one amplitude and one phase image which have improved contrast from thermal gradient changes across the surface due 
to the reduction of emissivity effects. The temperature of the samples at each pulse cycle is slightly different due to continuous pulsing of the thermal energy into the samples while collecting the data. Principal component analysis (PCA) is carried out independently on the matrix of the phase images and that of the amplitude images. Removing surface material to deface serial numbers exposes the zone of plastic strain or HAZ to the surface. These regions still extend to some depth below the surface with respect to stamping pressure and the amount of surface material removed during defacing. As a result, it is necessary to inspect both the amplitude images, which capture surface and near surface information, as well as phase images, which capture deeper lying information, in a bid to recover the defaced numbers.

The second step involves filtering the developed phase and amplitude images using a moving average filter as previously described. ${ }^{7}$ Principal component analysis is applied on these filtered images to localise the thermal gradient changes in the zone of plastic strain or HAZ into a single PCA score image or a subset of score images.

Principal component analysis of the amplitude and phase images from the different cycles will leverage on the anomalous heat propagation within the zone of plastic strain or the HAZ to project this region as a unique entity and reproduce the defaced number in a score image orthogonal to others. For the samples in this study, images are collected over 16 pulse cycles.

The output score images of each set (a set each for the amplitude and phase images) are visually inspected to determine the score image that best reproduces the defaced number. All 16 score images of the set within which a particular score image is adjudged to have best reproduced the number are used in the identification process irrespective of whether or not a number can visually be recognised in it.

For two of the samples used in this study (the gun barrel and needle holder), the setup is adjusted with the camera field of view focused to capture areas over two of the removed numbers. Reducing the area imaged in the experiments improves resolution thus helping to enhance the detail captured with more pixels across a single number. It also ensures that the areas captured have an even spread of the pulsed thermal energy from the laser beam to avoid possible lateral thermal gradients. Each dataset of thermal images captured is then digitally split to have datasets that are focused on the areas around each defaced number. An exception to this setup is the motorcycle specimen where it is not possible to identify the exact location of the defaced numbers. As such, the entire defaced area is captured in three sections and each section analysed to possibly recover some of the defaced numbers and use that as a guide to pinpoint the location of the defaced numbers.

\section{Number identification}

Identifying the number delineated by the local change in thermal gradient captured in respective score images involves extracting pertinent features from the images using PZMs and then comparing the features to the features of the PZMs of the reference number library using similarity measures.

\section{Pseudo Zernike moments}

Prior to extracting moment features, the score and library images are initially preprocessed into binary black-andwhite images and then resized into a larger square image of dimensions determined as shown in Equation 1.

$$
\left(N_{x}^{2}+N_{y}^{2}\right)^{1 / 2}
$$

where $N_{x}$ and $N_{y}$ are the image dimensions.

This larger image is created by padding each side of the binarised image with pixels of intensity 0 for black to create a new image of the larger dimensions. Resizing the image is done to ensure that the entirety of the original image is captured when the image is mapped into a unit circle required for computing PZMs. The resized images are then made scale and translation invariant as described in References 7, 9 and 15 by moving the centre of mass of objects in the image to the centre of the image and scaling the object size to a predetermined value (5000 object pixels for this study). These initial preprocessing steps ensures scale and translation invariance of all the images.

Calculating PZMs involves computing radial polynomials, basis functions and finally computation of moments by projecting an image onto the basis functions. This is similar to the computation of regular Zernike moments as used in previous work ${ }^{7}$ except in the computation of the real valued radial polynomials. These polynomials for PZMs are determined as shown in Equation 2.

$$
R_{n m}(\rho)=\sum_{s=0}^{n-m}(-1)^{s} * \frac{(2 n+1-s) !}{s !(n-|m|-s) !(n+|m|+1-s) !} * \rho^{n-s}
$$


where ns are a number of basis polynomials that define the order of PZMs and $m s$ are a positive or negative integer bound by only the constraint $|m| \leq n$. The extraction of PZMs requires determining an order or a range of orders of the polynomials at which the extracted features adequately describe the original image. A method applied in estimating this range is the image reconstruction error. ${ }^{15-17}$ This reconstruction error is determined by comparing the original input image to its reconstructed version from a set of PZMs at a specified pseudo Zernike polynomial order. The mean square reconstruction error $\varepsilon()$ between the original image and its reconstructed versions at increasing orders is used as a comparative measure, as described in Reference 7. Examples of the resulting cumulative sum plots are shown in Figure 4 for two library number images and a defaced number score image.

The figure shows the image representation ability of pseudo Zernike basis polynomials from 1 through 23 determined by the cumulative sum. Similar plots are obtained for other library and score images. The insets in the figure show the plots for the PZM vectors at basis polynomial orders of 10 and 15 , respectively, for a reference library number 2 .

The cumulative sum increases steadily until all major features have been captured and then it slowly increases, indicating little change in the mean square errors for consecutive orders and subtle features are now being included. Ultimately, there is a sudden increase at higher orders indicating all image features have been captured and higher order polynomials are adding non-feature related elements and distorting the reconstructed image. From the plot, this increase occurs at order 23 and thus, it can be deduced that beyond order 22, the reconstructed image is distorted and the PZM vectors will contain non-feature related elements that may impair the identification. The PZM feature vectors are developed by concatenating the PZMs at each order into a single vector. Vectors such as these from each image are used for comparing score images to reference library images.

\section{Similarity measures}

Identifying score images using the feature vectors obtained from PZMs is done using similarity measures. These measures are used to compare the feature vectors extracted from each score image to those from a reference library developed using Microsoft Word images of digits for 10 different font types as enumerated in Reference 7. Each score image is compared to the reference library images in this manner irrespective of whether or not a number can be visually recognised in it. The similarity measures used are listed alongside their equations in Table 1.

With multiple score images being compared using several sets of PZM vectors across 10 libraries using 15 similarity measures each, there is a need to determine a consensus across the resulting similarity values obtained. This consensus is achieved by exploiting high level data fusion to combine the similarity values into a single output, 8,18 The rules used are sum, geometric mean, harmonic mean, median, L-2 norm, L-1 norm, truncated

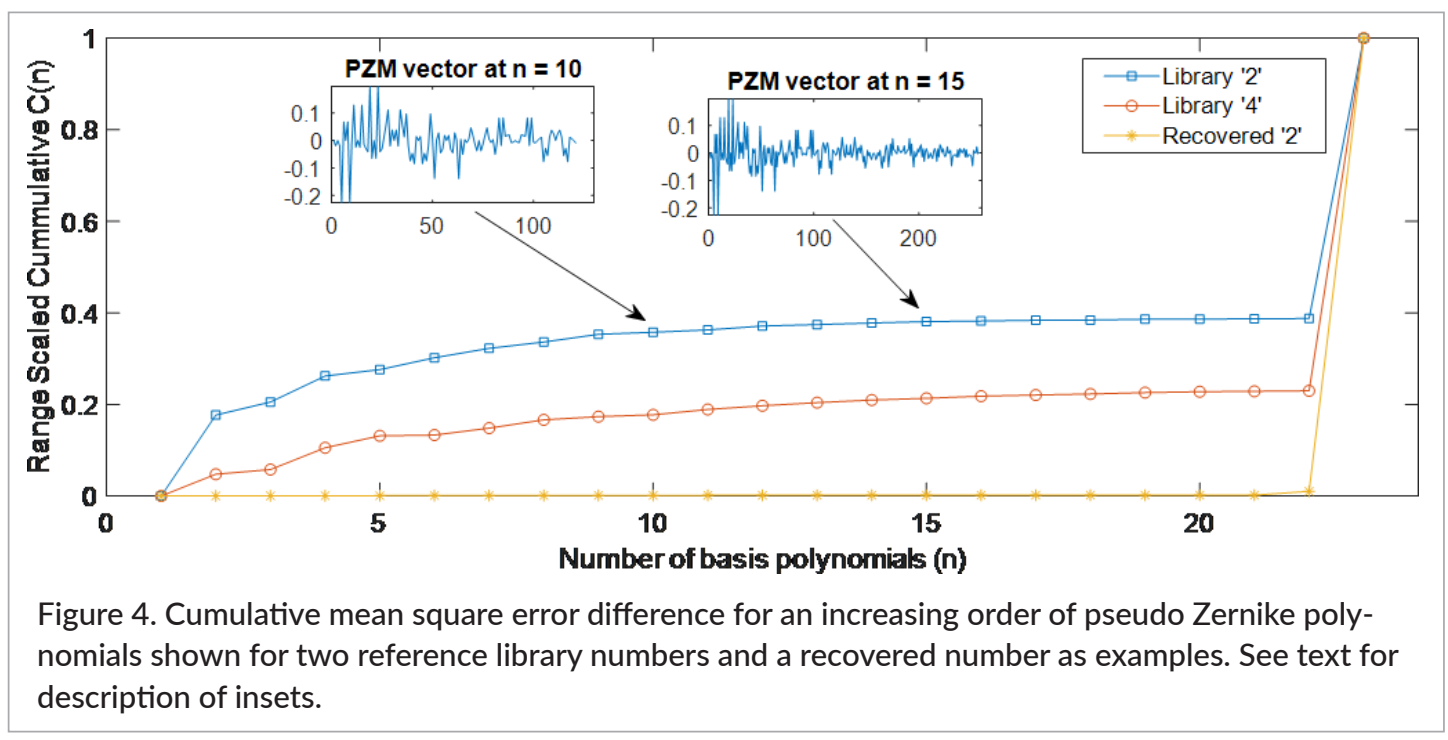


Table 1. Similarity measures used.

\begin{tabular}{|c|c|}
\hline Similarity measure ${ }^{a}$ & Equation $^{b}$ \\
\hline $\begin{array}{l}\text { Correlation coefficient } \\
\text { (2) }\end{array}$ & $C C=1-\frac{S_{I S}}{S_{1} S_{S}}$ \\
\hline Euclidean distance (2) & $E D=\sqrt{\left(x_{l}-x_{s}\right)\left(x_{l}-x_{s}\right)^{\top}}$ \\
\hline Angle (2) & $\cos \theta=1-\frac{\left|x_{1}^{\top} x_{s}\right|}{x_{1} x_{s}}$ \\
\hline Determinant (1) & $\operatorname{Det}=\left|\left(\begin{array}{l}x_{1}^{\top} \\
x_{s}^{\top}\end{array}\right)\left(\begin{array}{ll}x_{1} & x_{s}\end{array}\right)\right|$ \\
\hline $\begin{array}{l}\text { Procrustes analysis } \\
\text { (PA) (4) }\end{array}$ & $\mathrm{F}=\mathrm{F}_{\mathrm{sl}}-\mathrm{F}_{\mathrm{sSF}}$ \\
\hline Constrained PA (2) & $\mathrm{H}=\mathrm{H}_{\mathrm{sl}}-\mathrm{H}_{\mathrm{ssF}}$ \\
\hline $\begin{array}{l}\text { Mahalanobis distance } \\
\text { (2) }\end{array}$ & $M D=\sqrt{\left(x_{s}-x_{1}\right)^{\top} C_{s}^{+}\left(x_{s}-x_{l}\right)}$ \\
\hline
\end{tabular}

a alues in parenthesis indicate the number of variations

bsee Reference 7 for definitions of equations

$x_{1}$ feature vector of reference library image

$\mathrm{x}_{\mathrm{s}}$ feature vector of score image

$F$ Frobenius norm of the matrix

geometric mean and truncated harmonic mean. Most rules are applied twice, once to raw values and again to rank values. To obtain rank values, the raw similarity merit values obtained by comparing a score image to each of the possible library images are replaced by ranks from lowest to highest with lowest indicating smallest value as defined in Reference 7.

The identification of the defaced numbers is achieved by using two methods to obtain consensus from the 14 fusion rules used. These methods are majority vote and arithmetic sum. The majority vote consensus rule provides the numerical rank proposed by more than half of the fusion rules ( 8 out of 14 fusion rules in this study) in comparing the score images to the reference library images of a particular reference library number. The arithmetic sum adds up the numerical ranks for a particular reference library digit as adjudged by each fusion rule. The unknown sample (score images) is classified to the number digit with the lowest values in both instances (rank 1 for majority vote and minimum value for sum). A value "NC" is assigned if no particular rank has a majority across the fusion rules. A detailed description of these measures and how they are obtained and used can also be found in Reference 7 .

\section{Algorithms}

Algorithms for lock-in analysis, PCA, PZMs, similarity measures and fusion rules were written by the authors using MATLAB 9.2.

\section{Results and discussion}

The initial experiments employing this process, as reported previously, ${ }^{7}$ used a lock-in frequency of $0.05 \mathrm{~Hz}$ to modulate the thermal energy input into the sample. The results indicated $0.05 \mathrm{~Hz}$ to be a satisfactory modulation frequency for this process. In extending the testing to three other modulation frequencies, $1 \mathrm{~Hz}, 0.125 \mathrm{~Hz}$ and $0.03125 \mathrm{~Hz}$, this study tries to examine the possibility of utilising thermal images captured at several modulation frequencies in the recovery and classification process to eliminate the necessity of selecting an optimal frequency for each sample.

\section{Number recovery}

Gun barrel

Principal component analysis is carried out independently on the datasets of phase images and amplitude images developed from thermal images collected at each of the modulation frequencies. Respective score images are visually inspected to determine if phase or amplitude score images best reproduce the defaced number at a modulation frequency. For the area around the first defaced number on the gun barrel, a visual examination of the score images indicated that at all four modulation frequencies, the phase images better captured the thermal gradient variations within the zone of plastic strain to characterise the number. Figures 5 a to 5 e show the PCA score images for this area both before and after defacing. The defaced number is a 1 and shows up in the first PC as expected before defacing. However, after defacing, the score images with the recovered number are at higher PCs for each of the different modulation frequencies tested. This can be attributed to the phase shift due to thermal gradient differences within the defaced region accounting for a very small percentage of the variation compared to other thermal features within the thermal depth range.

From these images, it can be inferred that all four modulation frequencies could possibly induce adequate thermal gradients that will reproduce the number in the zone of plastic strain with varying degrees of clarity. 


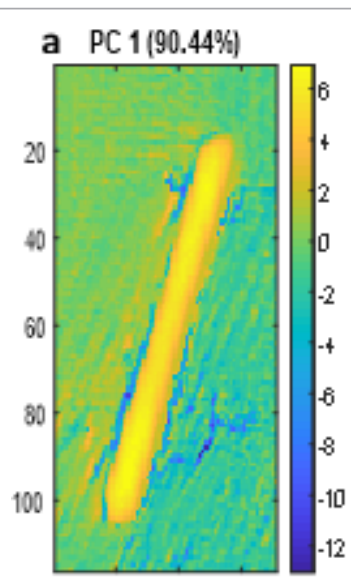

$20 \quad 40 \quad 60$
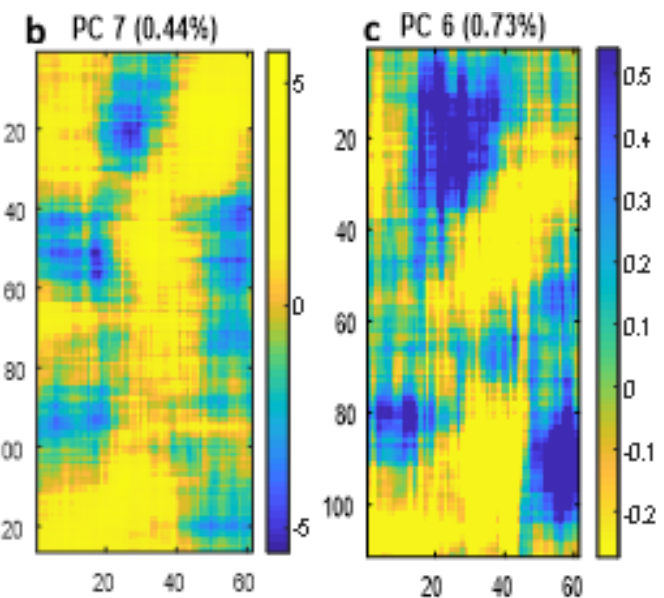
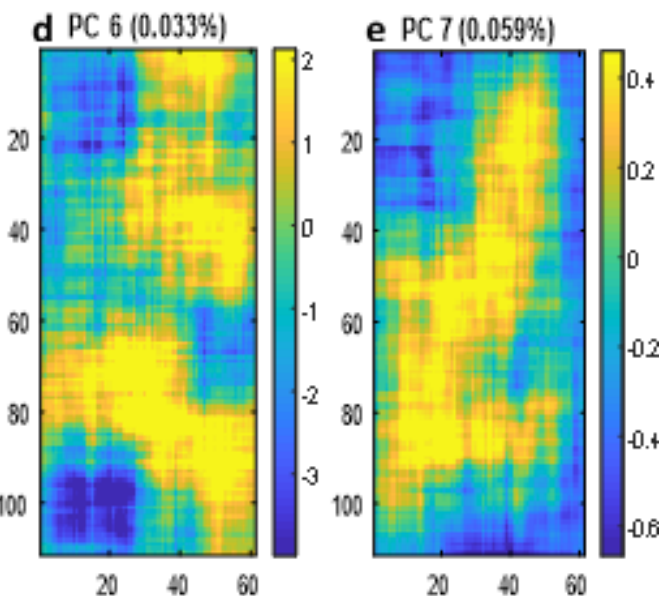

Figure 5. Gun barrel score images of a) clean 1 before defacing, and after defacing at b) $1 \mathrm{~Hz}, \mathrm{c}) 0.125 \mathrm{~Hz}, \mathrm{~d}) 0.05 \mathrm{~Hz}$ and e) $0.03125 \mathrm{~Hz}$.

Similarly, for the second defaced number, PCA is carried out independently on the matrices of the phase images and the amplitude images. Respective score images are visually inspected to determine which set best reproduces the defaced number at a modulation frequency. This visual examination showed that the phase images better characterised the thermal gradient changes indicative of the zone of plastic strain for frequencies $1 \mathrm{~Hz}$, $0.05 \mathrm{~Hz}$ and $0.03125 \mathrm{~Hz}$, and the amplitude images for $0.125 \mathrm{~Hz}$. As shown in Figures 6a to 6e, the number 2 showed up in PC 1 before defacing. After defacing, the zone of plastic strain characterising the defaced number is again captured in higher PCs for each of the different modulation frequencies used.

Summarily, it can be deduced that the four modulation frequencies used in the experiments are adequate for collecting thermal images to capture distinctive thermal gradients in the zones of plastic strain. However, there can be differences in the respective score image quality relative to modulation frequency due to differences in the depth of defacing from the force used in the stamping. Principal component analysis was tested using both phase and amplitude images together but the resulting score images (shown in the Appendix) were not as clearly defined as those developed by analysing each set separately.

\section{Laser engraved needle holder}

The same process was replicated for the laser engraved needle holder with the defaced area pulsed with thermal energy at four modulation frequencies. A visual inspection of the developed score images from the phase

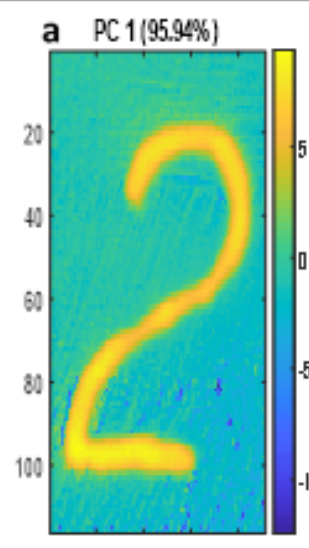

$2040 \quad 6080$

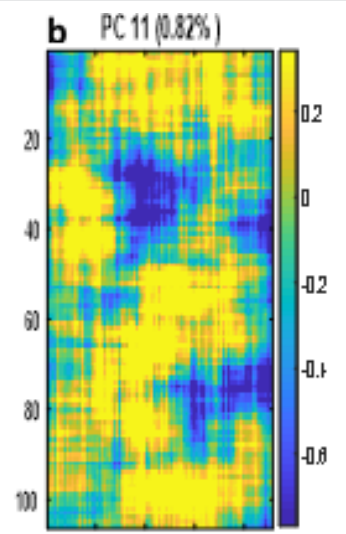

20. 4) 6) 8?

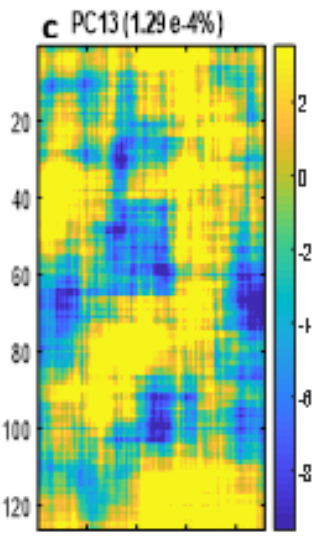

$20406080 \times 10^{-3}$

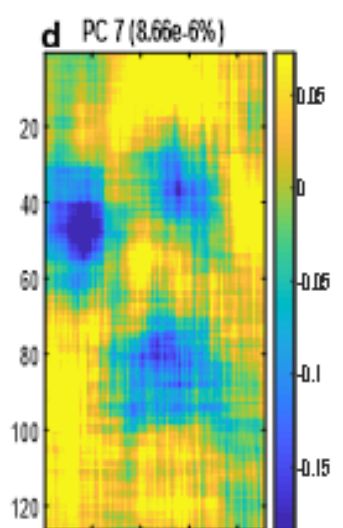

$20 \quad 40 \quad 60 \quad 80$

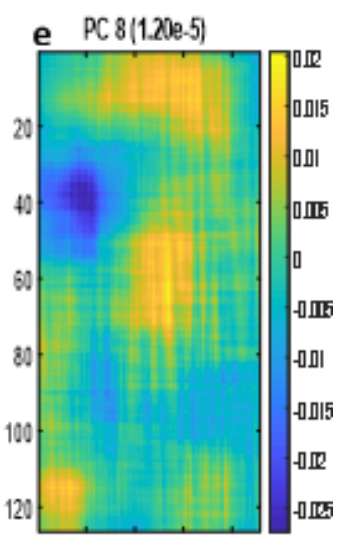

$20 \quad 40 \quad 6080$

Figure 6. Gun barrel score images of a) clean 2 before defacing, and after defacing at b) $1 \mathrm{~Hz}, \mathrm{c}) 0.125 \mathrm{~Hz}, \mathrm{~d}) 0.05 \mathrm{~Hz}$ and e) $0.03125 \mathrm{~Hz}$. 
and amplitude images at each modulation frequency showed the amplitude score images best characterised the thermal gradient differences in the HAZ at all four modulation frequencies for the defaced number 0 . For the defaced number 3 , visual inspection identified amplitude score images at all four modulation frequencies as best representing the defaced number. Figure 7 shows the score images of the defaced number 0 , for the four modulation frequencies. Figure 8 similarly shows the score images from different modulation frequencies for the defaced number 3 .

As with the gun barrel, score images with the recovered digits are at higher PCs explaining small amounts of variance for the four pulsing frequencies. From the resulting score images of both samples, it can again be inferred that all four modulation frequencies adequately captured the thermal gradient changes. These results further suggest that all four modulation frequencies can induce adequate thermal gradients. However, due to variations in depths of the deformed regions from the stamping or laser engraving process as well as in the defacing of the different areas, there may be small differences in the quality of a recreated number in respective score images at different frequencies. Thus, it is probably best to analyse thermal images at several modulation frequencies and then utilise the resulting score images from all the frequencies in identifying the defaced numbers.
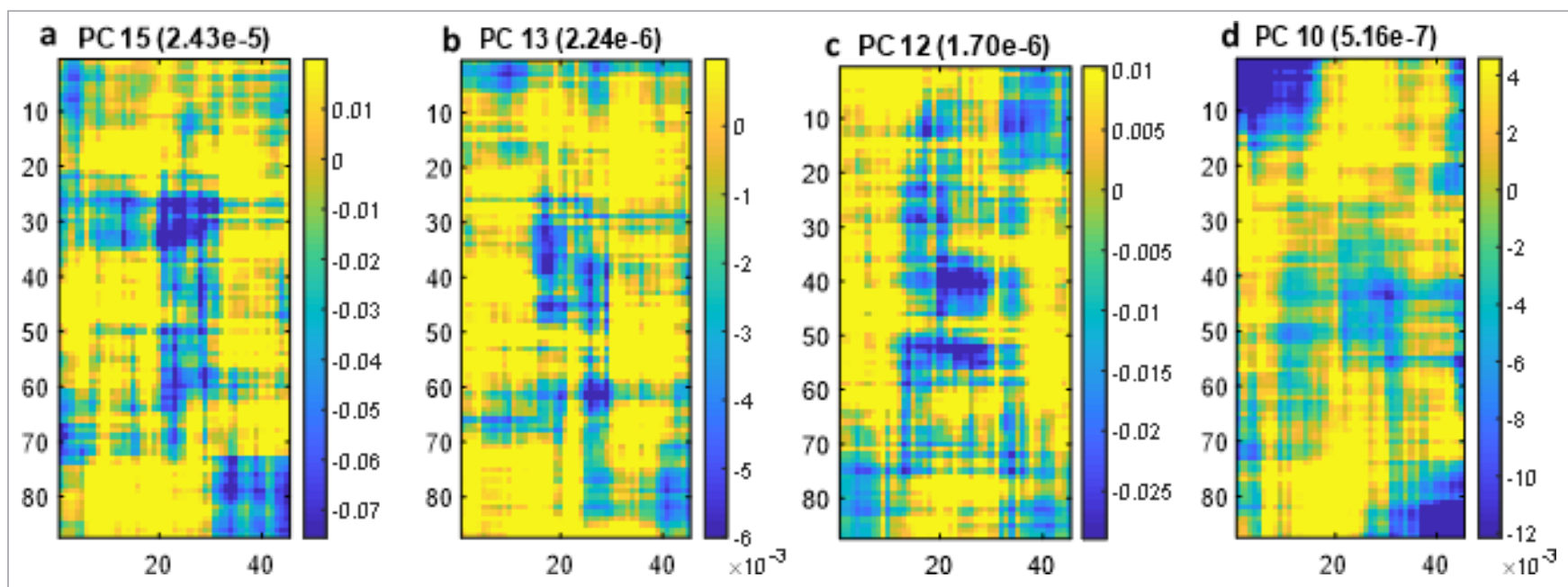

Figure 7. Needle holder score images of defaced area around 0 at a) $1 \mathrm{~Hz}$, b) $0.125 \mathrm{~Hz}$, c) $0.05 \mathrm{~Hz}$ and d) $0.03125 \mathrm{~Hz}$.

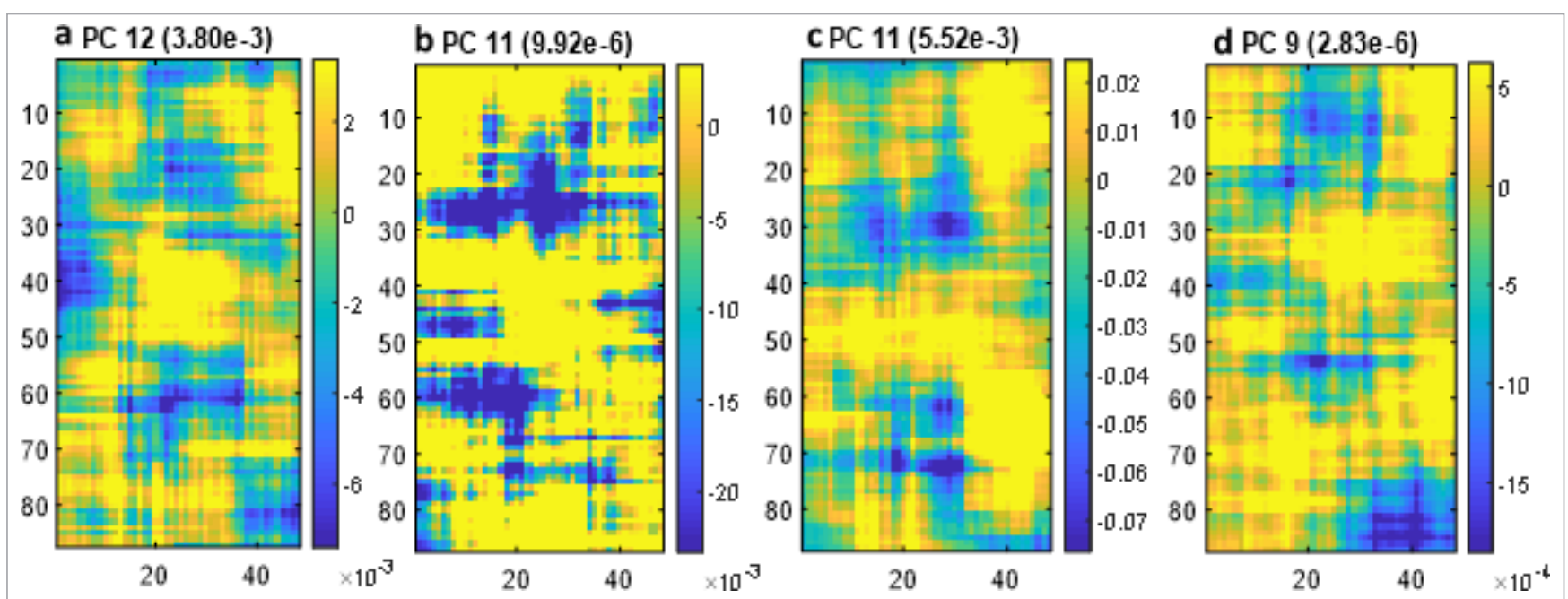

Figure 8. Needle holder score images of defaced area around number 3 at a) $1 \mathrm{~Hz}$, b) $0.125 \mathrm{~Hz}, \mathrm{c}) 0.05 \mathrm{~Hz}$ and d) $0.03125 \mathrm{~Hz}$. 
Yamaha motorcycle

The motorcycle fork had the VIN completely removed but the VIN stamped into the engine block only had a few numbers defaced. From the intact numbers on the engine block, it was determined that the VIN numbers are 5-2-5-1-[]-0-[]-4-1 with the square brackets indicating the completely defaced numbers. Assuming that the motor was original, knowledge of some of the serial numbers allows for the evaluation of the identification process using only the defaced fork numbers.

An initial challenge was to narrow down the locations of the stamped fork numbers. To narrow down the locations, the entire defaced area was divided into three sections as shown in Figure 9. The developed process using LIT and PCA was carried out on each section to investigate the possibility of locating regions where a number may have been and use this as a template to locate the positions of other numbers in the area. This process was carried out on the thermal images collected at each of the modulation frequencies tested.

A score image of the middle section in Figure 9 is shown in Figure 10. This score image developed from the thermal images collected at $0.05 \mathrm{~Hz}$ shows what appears to be parts of numbers allowing specific locations to be identified. The centre to centre distance between each of the possible numbers was determined for use as a guide to possible positions of other numbers. This distance is approximately 125 pixels wide. The bounded regions (white boxes in Figure 10) were extracted from the original dataset and independently re-analysed by PCA to characterise the local zones of plastic strain and recover the defaced numbers within.

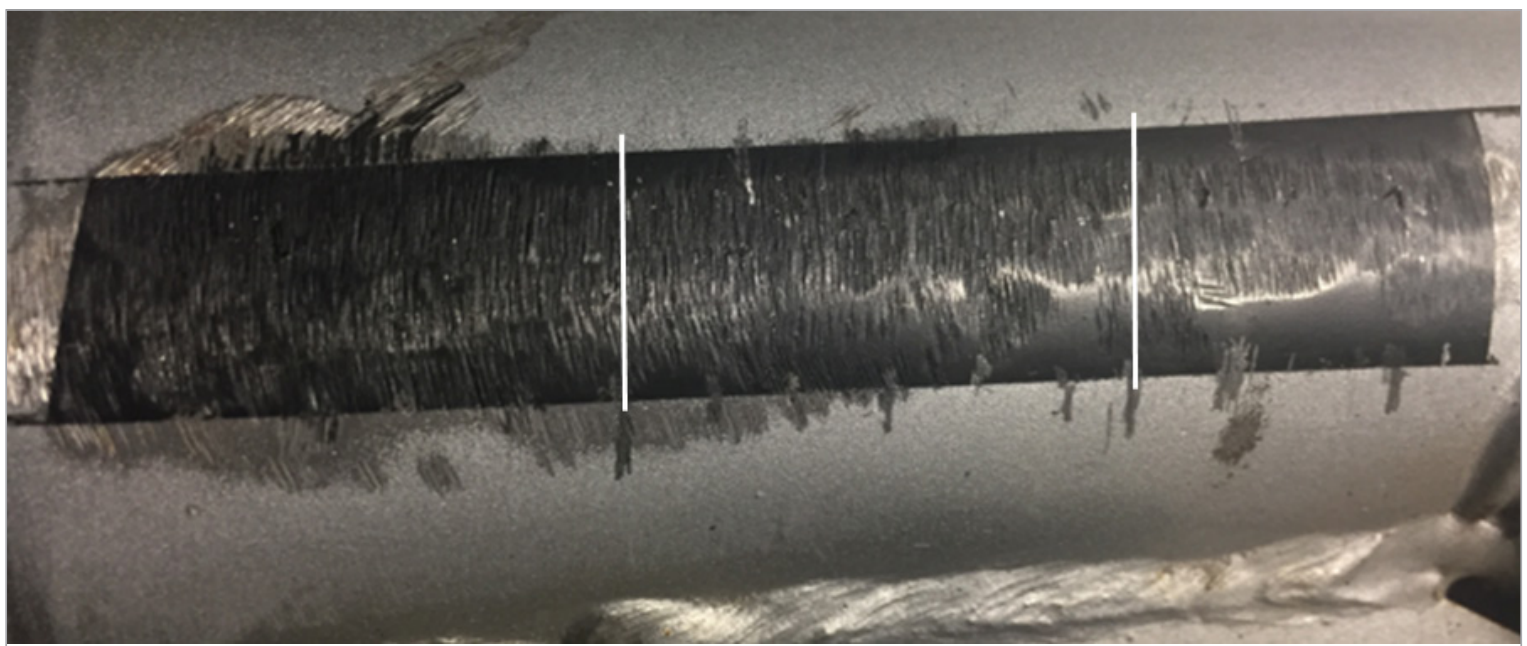

Figure 9. Defaced motorcycle fork painted with India ink (white lines demarcate the sections individually imaged and analysed).

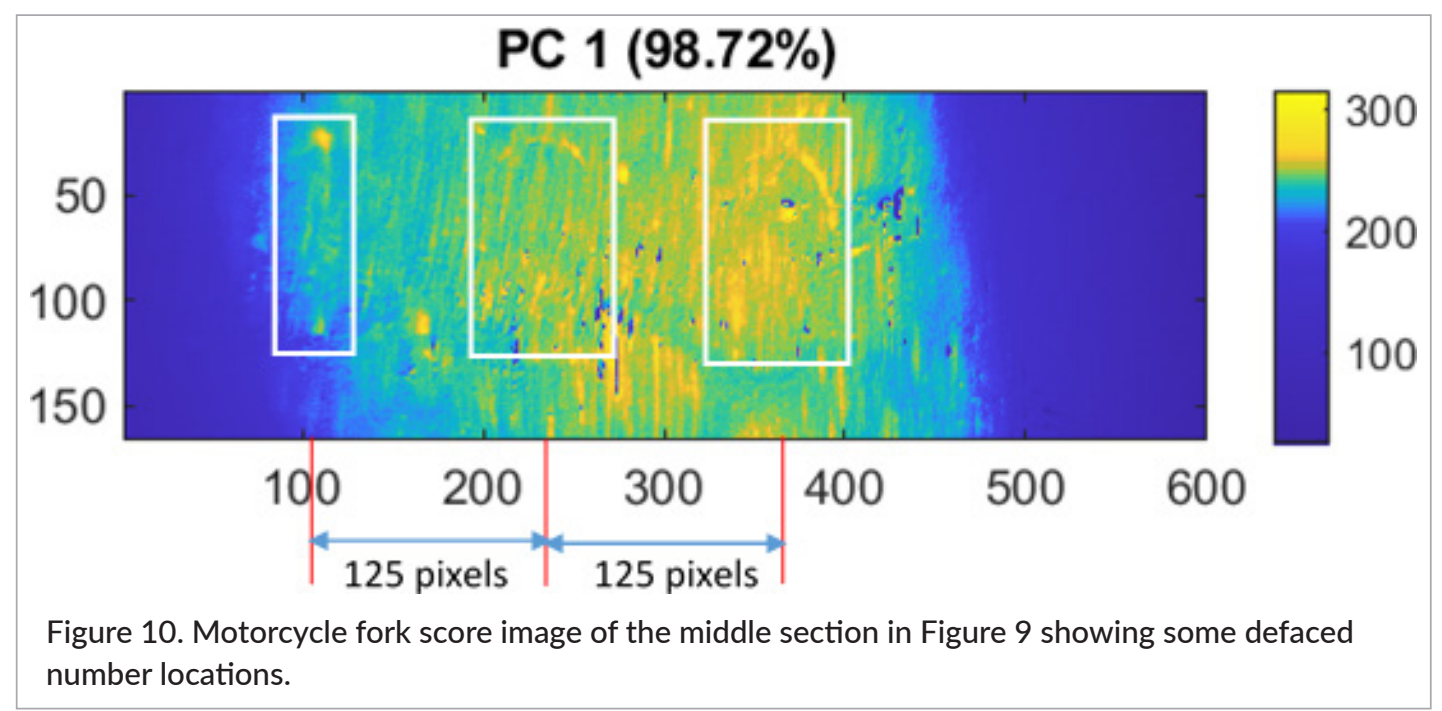


A similar PCA assessment of the first section produced an area in a PC score image that could conceivably be identified as the zone of plastic strain beneath a defaced number. This area was extracted as a region of interest within the bounds of the white box shown on the image in Figure 11 and re-analysed independently. The centre to centre distance previously determined from the assessment of the middle section was used to determine the centre of the next two numbers in the image to form the regions of interest (red boxes in Figure 11) to be re-analysed independently by PCA.

The initial PCA assessment of the third section from Figure 9 can be seen in Figure 12. A characteristic shape that may represent a local zone of plastic strain was identified and the region of interest was localised (white box in Figure 12). This localised area was used to determine the possible locations of other numbers in this section (red boxes in Figure 12) via the centre to centre distance determined from the defaced numbers in Figure 10.

Each of these localised regions of interest were independently analysed using PCA to characterise and enhance the local thermal gradients around the defaced numbers. Both phase and amplitude images developed at each modulation frequency were separately analysed and inspected to visually determine which best characterised the zone of plastic strain captured in the thermal images. This proved to be the phase images for $1 \mathrm{~Hz}, 0.125 \mathrm{~Hz}$ and $0.05 \mathrm{~Hz}$, and amplitude images for $0.03125 \mathrm{~Hz}$ for the first number 5. For the defaced number 2 , the amplitude score images best characterised the zone of plastic strain at frequencies $1 \mathrm{~Hz}, 0.05 \mathrm{~Hz}$ and $0.03125 \mathrm{~Hz}$, and the phase score images at $0.125 \mathrm{~Hz}$. For the second number 5 , the amplitude score images best reproduced the defaced number at $1 \mathrm{~Hz}$, $0.125 \mathrm{~Hz}$ and $0.03125 \mathrm{~Hz}$, and the phase score images at $0.05 \mathrm{~Hz}$. For the first number 1, the phase score images better reproduced the defaced number at all four modulation frequencies. For the first unknown digit, the phase score images better characterised the defaced number at $1 \mathrm{~Hz}$ and the amplitude score images at the other three modulation frequencies. For the second number 0 , the amplitude score images better characterised the thermal gradient variations within the zone of plastic strain to reproduce the number at $1 \mathrm{~Hz}$, and the phase score images at the other three modulation frequencies. For the second unknown digit, the amplitude score images were deemed to better characterise the local change in thermal gradient indicative of the zone of plastic strain at $1 \mathrm{~Hz}, 0.125 \mathrm{~Hz}$ and $0.03125 \mathrm{~Hz}$, and the phase score images at $0.05 \mathrm{~Hz}$.
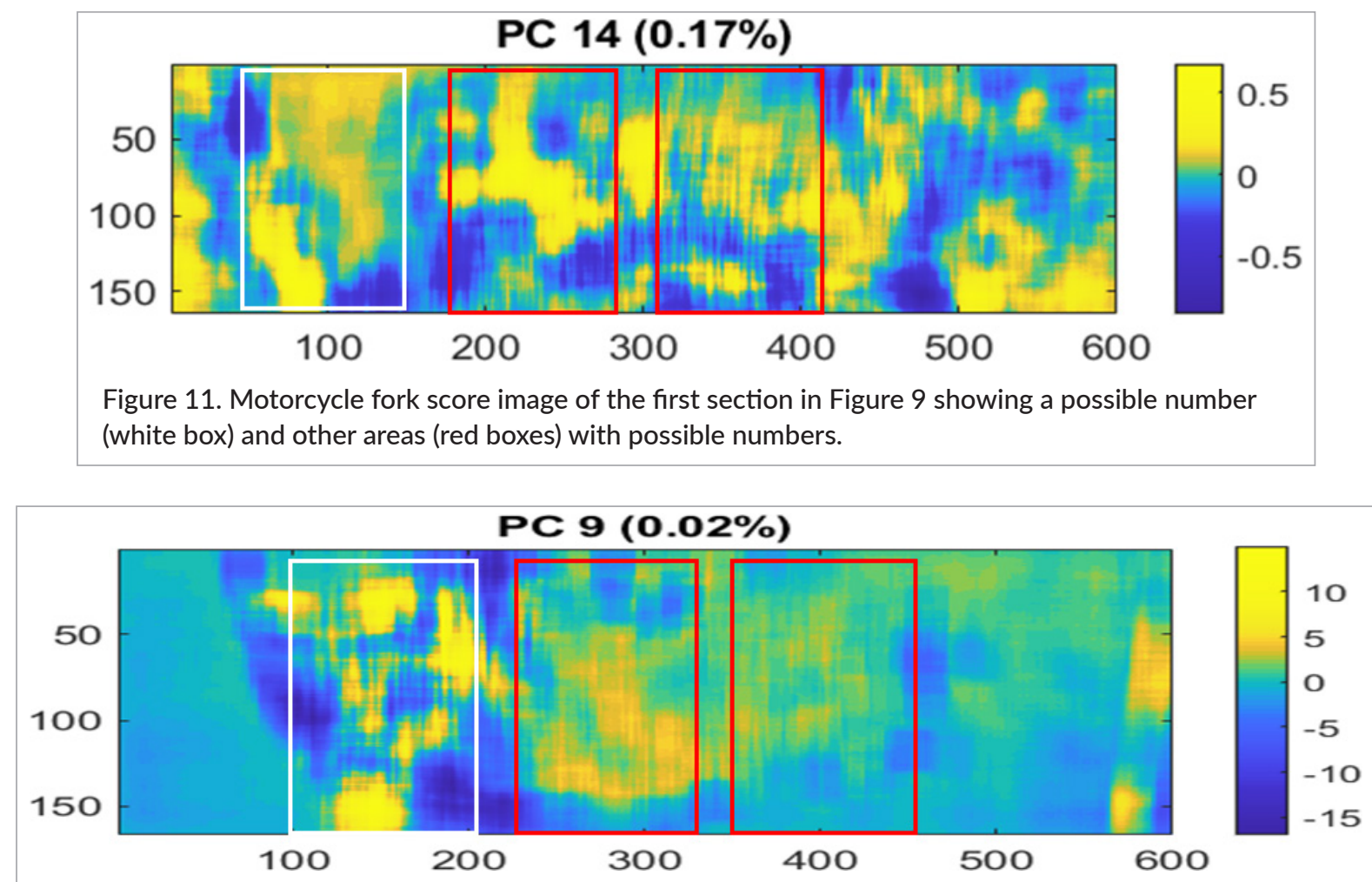

Figure 12. Motorcycle fork score image of the third section in Figure 9 showing a possible number (white box) and other areas (red boxes) with possible numbers. 
The phase images also better characterised the zone of plastic strain at $1 \mathrm{~Hz}, 0.125 \mathrm{~Hz}$, and the amplitude images at $0.05 \mathrm{~Hz}$ and $0.03125 \mathrm{~Hz}$ for the defaced number 4. For the second number 1 , the amplitude images better characterised the difference in thermal gradients at $1 \mathrm{~Hz}, 0.05 \mathrm{~Hz}$ and $0.03125 \mathrm{~Hz}$, and the phase images at $0.125 \mathrm{~Hz}$. Figure 13 shows a selected score image for each of the different areas. These images selected by visual inspection of all 16 score images developed for each local region of interest across each of the four modulation frequencies (a total of 64 comparisons), show enough detail to mark out a possible number. As with the gun barrel and needle holder, only one score image shows enough of such details with the other score images only partially characterising the number. The numbers listed in the title for each score image are the known stamped numbers from the engine block. The numbers also defaced on the engine block are labelled unknown.

The images shown in Figure 13 are a selection of score images deemed to best characterise the zone of plastic strain and reproduce each of the defaced numbers. Although only one score image at a single modulation frequency is shown for each defaced number, thermal images collected at all four modulation frequencies capture enough of the thermal gradient to characterise the zone of plastic strain. However, there are slight differences in the quality of the characterised zone of plastic strain owing to the local conditions of each of the defaced areas. Such discrepancies in the quality of the score images lend credence the proposed process of collecting data at several modulation frequencies, avoiding any need to select a single modulation frequency that will be adequate for any defaced surface under investigation.

From the score images of the defaced areas shown in this section, PCA appears to adequately capture the variation in thermal gradient to allow for the recreation of the defaced numbers. However, as previously enumerated, several factors local to each tested area affect the quality of the score images and make visual recognition of the numbers difficult. This difficulty in visual identification, highlights the necessity of confirming the identities of the defaced numbers in the score images.

\section{Number identification}

All score images developed from each of the four modulation frequencies tested are used in the identification process, eliminating any need to preselect a particular frequency to use. As previously described, all the simi- larity merit values obtained for the comparison of a set of score images to a single reference library digit are fused into a single representative value using the different fusion rules. These fused values are ranked with respect to the fused values for all the different digits from lowest to highest. These ranks indicate how closely the score images of the defaced numbers are to the particular reference library digit relative to all the other digits. The defaced number is matched to the reference library number that is most consistently ranked lowest (rank =1) across the fusion rules (eight of the fourteen rules, i.e. majority vote) and whose sum of the ranked fusion rules is lowest. Higher resulting rank values for the reference library numbers indicate an increasingly worse fit of the particular digit to the score images. Both the majority vote and sum rule have to be in unison in identifying a number or the identification will be deemed inconclusive.

\section{Gun barrel}

Tables 2 and 3 show the results for the identification of the defaced numbers on the gun barrel for the methods used to obtain consensus (majority vote and sum, respectively) across the fusion rules. The shaded boxes identify the digit that was determined to be the best match for the score images of each tested area. The value in each cell corresponds to the consensus rank across all the fusion rules and the sum of the ranks across all the fusion rules for majority vote and sum, respectively. From these tables, the consensus results positively match the defaced numbers to their contemporaries in the reference library. This demonstrates the importance of such an identification protocol. Although the score images of the defaced numbers do not enhance the thermal gradients enough to visually identify the numbers, any uncertainties as to their identities are eliminated by such verification.

\section{Needle holder}

Tables 4 and 5 show the results of obtaining a consensus of all the resulting fusion values for the defaced numbers (numbers 0 and 3 ) on the laser engraved needle holder using both methods (majority vote and sum, respectively). From these tables, the consensus results positively match the defaced numbers to the respective reference library images. This identification protocol helps to eliminate uncertainties in the identities of the defaced numbers as the score images that characterise the zone of plastic strain do not have the visual quality to allow for objective identification of the number. 


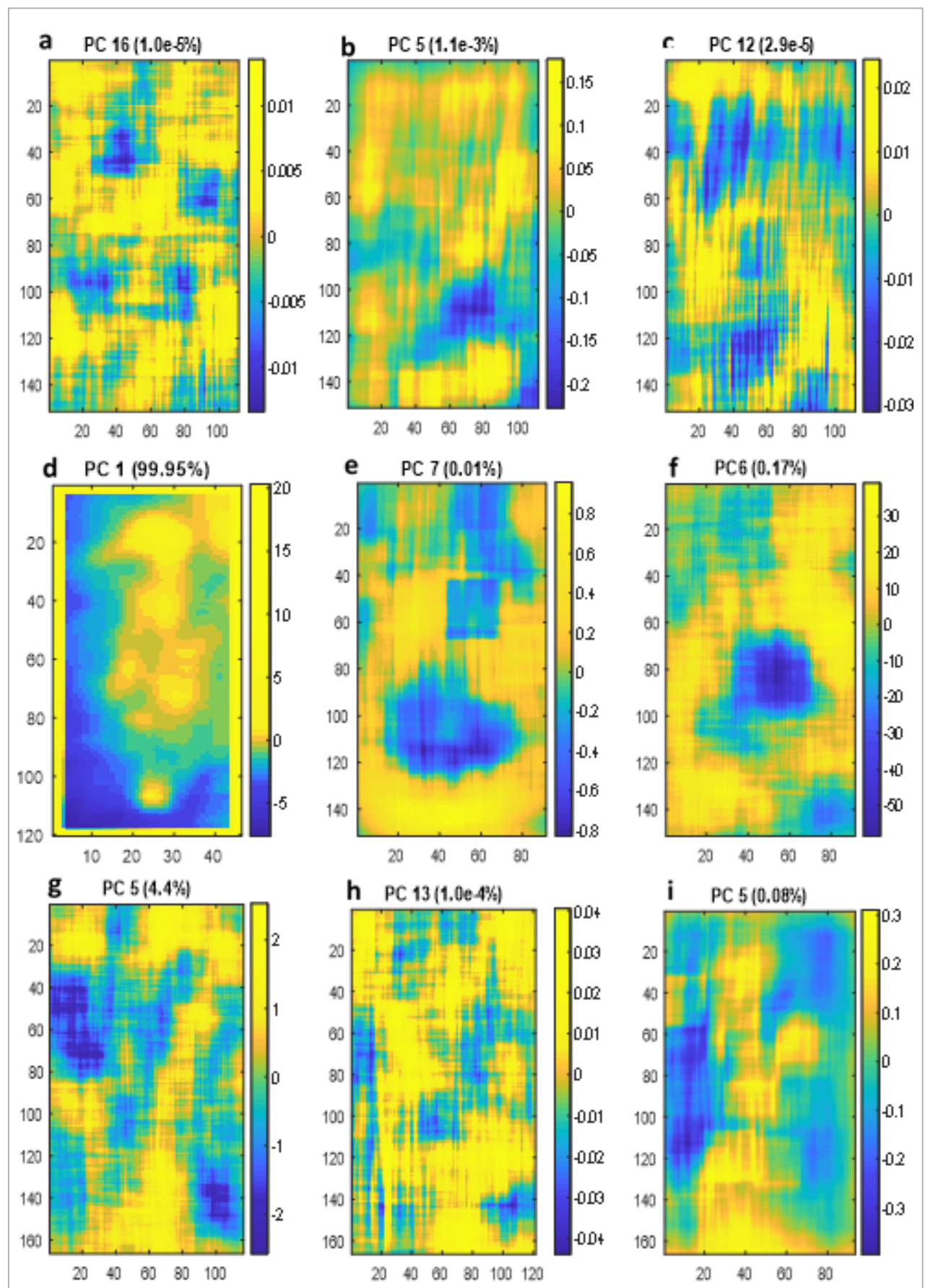

Figure 13. Motorcycle fork score images showing the recovered VIN numbers a) 5 $(0.03125 \mathrm{~Hz})$, b) $2(0.03125 \mathrm{~Hz})$, c) $5(0.03125 \mathrm{~Hz})$, d) $1(0.05 \mathrm{~Hz})$, e) unknown $(0.05 \mathrm{~Hz})$, f) $0(0.05 \mathrm{~Hz}), \mathrm{g})$ unknown $(0.05 \mathrm{~Hz}), \mathrm{h}) 4(0.05 \mathrm{~Hz})$ and i) $1(0.05 \mathrm{~Hz})$.

Motorcycle VIN

Tables 6 and 7 show the results of the consensus of identification for the defaced numbers on the motorcycle fork using majority vote and sum, respectively, as consensus methods. Shown in these tables are the consensus ranks for the reference library number matched to the score images of each tested defaced area. Comparing these results to the known numbers from the engine block, it is seen that the defaced serial numbers were accurately identified.

From the results in Tables 2 to 7, it can be deduced that identifying defaced numbers using score images 
Table 2. Majority vote fusion of similarity measure values for defaced areas on gun barrel.

\begin{tabular}{|c|c|c|}
\hline \multirow{2}{*}{$\begin{array}{c}\text { Ref. library } \\
\text { numbers }\end{array}$} & \multicolumn{2}{|c|}{ Defaced numbers } \\
\cline { 2 - 3 } & 1 & 2 \\
\hline 0 & 4 & 3 \\
\hline 1 & 1 & 9 \\
\hline 2 & 2 & 1 \\
\hline 3 & 6 & 6 \\
\hline 4 & 9 & 10 \\
\hline 5 & $N C^{*}$ & 2 \\
\hline 6 & 7 & 5 \\
\hline 7 & 8 & 7 \\
\hline 8 & 5 & 4 \\
\hline 9 & 10 & 8 \\
\hline
\end{tabular}

${ }^{*} \mathrm{NC}$ indicates there was no consensus rank for the digit across the fusion rules

Table 4. Majority vote fusion of similarity measure values for defaced areas on needle holder.

\begin{tabular}{|c|c|c|}
\hline \multirow{2}{*}{$\begin{array}{c}\text { Ref. library } \\
\text { numbers }\end{array}$} & \multicolumn{2}{|c|}{ Defaced numbers } \\
\cline { 2 - 3 } & 0 & 3 \\
\hline 0 & 1 & 6 \\
\hline 1 & 2 & 5 \\
\hline 2 & 6 & 2 \\
\hline 3 & 4 & 1 \\
\hline 4 & 7 & 9 \\
\hline 5 & 3 & 4 \\
\hline 6 & 8 & 10 \\
\hline 7 & 10 & 3 \\
\hline 8 & 5 & 8 \\
\hline 9 & 9 & $\mathrm{NC}^{*}$ \\
\hline
\end{tabular}

${ }^{*} \mathrm{NC}$ indicates there was no consensus rank for the digit across the fusion rules

developed from thermal images collected at multiple modulation frequencies is feasible. Using thermal images collected at several modulation frequencies negates the need to select a particular modulation frequency to use in the identification process, and using all the score images avoids any need to select a particular one to use in identifying the defaced number. However, the selection of a set of score images based on either phase or amplitude images based on visual inspection is still necessary.
Table 3. Sum fusion of similarity measure values for defaced areas on gun barrel.

\begin{tabular}{|c|c|c|}
\hline \multirow{2}{*}{$\begin{array}{c}\text { Ref. library } \\
\text { numbers }\end{array}$} & \multicolumn{2}{|c|}{ Defaced numbers } \\
\cline { 2 - 3 } & 1 & 2 \\
\hline 0 & 55 & 81 \\
\hline 1 & 26 & 38 \\
\hline 2 & 28 & 14 \\
\hline 3 & 85 & 59 \\
\hline 4 & 124 & 67 \\
\hline 5 & 31 & 37 \\
\hline 6 & 93 & 126 \\
\hline 7 & 114 & 110 \\
\hline 8 & 74 & 98 \\
\hline 9 & 140 & 140 \\
\hline & & \\
\hline
\end{tabular}

Table 5. Sum fusion of similarity measure values for defaced areas on needle holder.

\begin{tabular}{|c|c|c|}
\hline \multirow{2}{*}{$\begin{array}{c}\text { Ref. library } \\
\text { numbers }\end{array}$} & \multicolumn{2}{|c|}{ Defaced numbers } \\
\cline { 2 - 3 } & 0 & 3 \\
\hline 0 & 14 & 90 \\
\hline 1 & 30 & 70 \\
\hline 2 & 84 & 26 \\
\hline 3 & 56 & 16 \\
\hline 4 & 104 & 126 \\
\hline 5 & 40 & 50 \\
\hline 6 & 106 & 140 \\
\hline 7 & 140 & 48 \\
\hline 8 & 70 & 110 \\
\hline 9 & 126 & 94 \\
\hline
\end{tabular}

\section{Conclusion}

A non-destructive process is proposed for use as an alternative to established destructive processes for the restoration of defaced serial numbers. This process involves utilising lock-in infrared thermography and PCA to capture and enhance the unique thermal gradients within defaced areas to delineate the obliterated numbers in score images. Invariant features are extracted from the developed score images using PZMs 
Table 6. Majority vote fusion of similarity measure values for recovered numbers on the motorcycle fork.

\begin{tabular}{|c|r|r|r|r|r|r|r|r|r|}
\hline \multirow{2}{*}{$\begin{array}{c}\text { Ref. library } \\
\text { numbers }\end{array}$} & \multicolumn{9}{|c|}{ Defaced numbers } \\
\cline { 2 - 11 } & 5 & 2 & 5 & 1 & 0 & 0 & 7 & 4 & 1 \\
\hline 0 & 5 & 7 & $N C^{*}$ & 7 & 1 & 1 & 8 & 8 & $N C^{*}$ \\
\hline 1 & $N C^{*}$ & 4 & 3 & 1 & 7 & 9 & $N C^{*}$ & 4 & 1 \\
\hline 2 & 2 & 1 & $N C^{*}$ & 2 & 4 & 4 & $N C^{*}$ & $N C^{*}$ & 2 \\
\hline 3 & 3 & 3 & 7 & 4 & 6 & 6 & 5 & $N C^{*}$ & 4 \\
\hline 4 & $N C^{*}$ & 10 & 10 & 6 & $N C^{*}$ & 10 & 9 & 1 & 5 \\
\hline 5 & 1 & 2 & 1 & $N C^{*}$ & $N C^{*}$ & 3 & 4 & $N C^{*}$ & 3 \\
\hline 6 & $N C^{*}$ & 8 & 9 & 9 & 5 & 5 & 10 & 7 & 9 \\
\hline 7 & $N C^{*}$ & 6 & 5 & 5 & 10 & $N C^{*}$ & 1 & 9 & 6 \\
\hline 8 & 4 & 5 & 6 & $N C^{*}$ & $N C^{*}$ & 2 & 6 & 5 & 7 \\
\hline 9 & 10 & 9 & 8 & 10 & $N C^{*}$ & 7 & 7 & 10 & 10 \\
\hline
\end{tabular}

*NC indicates there was no consensus rank for the digit across the fusion rules

Table 7. Sum fusion of similarity measure values for recovered numbers on the motorcycle fork.

\begin{tabular}{|c|c|c|c|c|c|c|c|c|c|}
\hline \multirow{2}{*}{$\begin{array}{c}\text { Ref. library } \\
\text { numbers }\end{array}$} & \multicolumn{9}{|c|}{ Defaced numbers } \\
\hline & 5 & 2 & 5 & 1 & 0 & 0 & 7 & 4 & 1 \\
\hline 0 & 73 & 95 & 56 & 104 & 22 & 24 & 103 & 106 & 115 \\
\hline 1 & 77 & 55 & 38 & 24 & 104 & 124 & 34 & 53 & 18 \\
\hline 2 & 28 & 14 & 30 & 26 & 49 & 50 & 28 & 23 & 26 \\
\hline 3 & 54 & 45 & 90 & 57 & 87 & 78 & 68 & 81 & 54 \\
\hline 4 & 130 & 140 & 134 & 84 & 111 & 128 & 126 & 20 & 73 \\
\hline 5 & 14 & 28 & 22 & 36 & 27 & 34 & 56 & 46 & 42 \\
\hline 6 & 102 & 112 & 127 & 124 & 73 & 77 & 140 & 101 & 120 \\
\hline 7 & 108 & 87 & 70 & 67 & 138 & 126 & 24 & 126 & 83 \\
\hline 8 & 50 & 68 & 86 & 108 & 43 & 32 & 87 & 74 & 99 \\
\hline 9 & 134 & 126 & 117 & 140 & 116 & 97 & 104 & 140 & 140 \\
\hline
\end{tabular}

and compared to a reference library to identify the defaced numbers. The presented process is successfully tested on several specimens including mechanically stamped samples and a laser engraved sample. With the successful recovery of defaced numbers on the samples tested, this non-destructive process shows potential for use as an alternative to or as a precursor to the established methods for both mechanically stamped and laser engraved specimens. The process is also quite flexible with several independent parts including the thermographic technique (LIT), image analysis (PCA), feature extraction (PZM) and similarity measures for image identification. Each of these parts can conceiv- ably be replaced with alternative methods, allowing for further testing and possible improvement of the defaced number recovery and identification process.

\section{Acknowledgements}

This work was supported by the National Institute of Justice, Office of Justice Programs (grant numbers 2013R2-CX-K012 and 2015-R2-CX-0017). The opinions, findings and conclusions or recommendations expressed in this exhibition are those of the authors and do not necessarily reflect those of the Department of Justice. 
We would also like to thank Royce Martin from ISU Electronic Services and Miles Whiting from Machining Services for their help with the equipment and sample fabrication.

\section{Declarations of interest}

Patent pending

\section{References}

1. D.E. Polk and B. Giessen, "Metallurgical aspects of serial number recovery", AFTE J. 21(2), 174 (1989).

2. H. Katterwe, "Restoration of serial numbers", in Forensic Investigation of Stolen-Recovered and Other Crime-Related Vehicles, Ed by E. Stauffer and M.S. Bonfanti. Elsevier, Ch. 7, p. 177 (2006). https://doi. org/10.1016/B978-012088486-5/50041-7

3. E. Stauffer and M.S. Bonfanti (Eds), Forensic Investigation of Stolen-Recovered and Other CrimeRelated Vehicles. Elsevier/Academic Press (2006). https://doi.org/10.1016/B978-0-12-088486-5. $\times 5030-3$

4. L. da Silva and P.A.M. dos Santos, "Recovering obliterated laser engraved serial numbers in firearms", Forensic Sci. Int. 179(2-3), e63 (2008). http://doi. org/10.1016/j.forsciint.2008.05.012

5. R.S. Treptow, Methods for the Restoration of Obliterated Serial Numbers. National Institute of Justice (1977). https://www.ncjrs.gov/App/ Publications/abstract.aspx?ID=81771

6. D.M. Turley, "Restoration of stamp marks on steel components by etching and magnetic techniques", J. Forensic Sci. 32(3), 640 (1987). https://doi. org/10.1520/JFS12368J

7. I. Unobe, L. Lau, J. Kalivas, R. Rodriguez and A. Sorensen, "Restoration of defaced serial numbers using lock-in infrared thermography (part I)", J. Spectral Imaging 8, a19 (2019). https://doi. org/10.1255/jsi.2019.a19

8. E. Borràs, J. Ferré, R. Boqué, M. Mestres, L. Aceña and $\mathrm{O}$. Busto, "Data fusion methodologies for food and beverage authentication and quality assessment
- a review", Anal. Chim. Acta 891, 1 (2015). https:// doi.org/10.1016/J.ACA.2015.04.042

9. C.-H. Teh and R.T. Chin, "On image analysis by the methods of moments", IEEE Trans. Pattern Anal. Mach. Intell. 10(4), 496 (1988). https://doi. org/10.1109/34.3913

10. Y.-H. Pang, A.B.J. Teoh and D.C.L. Ngo, "A discriminant pseudo Zernike moments in face recognition", J. Res. Pract. Inf. Technol. 38(2), 197 (2006).

11. T. Xia, H. Zhu, H. Shu, P. Haigron and L. Luo, "Image description with generalized pseudo-Zernike moments", JOSA A 24(1), 50 (2007). https://doi. org/10.1364/JOSAA.24.000050

12. A. Killey and J.P. Sargent, "Analysis of thermal nondestructive testing", J. Phys. D. Appl. Phys. 22(1), 216 (1989). https://doi.org/10.1088/0022$3727 / 22 / 1 / 032$

13. H. Liu, Y. Wang, J. Liu and H. Gong, "Effect of modulation frequency on detecting defects of metal plates using infrared lock-in thermography", in 4th International Symposium on Advanced Optical Manufacturing and Testing Technologies: Optical Test and Measurement Technology and Equipment 7283, 72833M (2009). https://doi.org/10.1117/12.828780

14. O. Breitenstein, W. Warta and M. Langenkamp, Lock-in Thermography: Basics and Use for Evaluating Electronic Devices and Materials. Springer, Berlin/ Heidelberg (2010). https://doi.org/10.1007/978-3642-02417-7

15. A. Khotanzad and Y.H. Hong, "Invariant image recognition by Zernike moments", IEEE Trans. Pattern Anal. Mach. Intell. 12(5), 489 (1990). https://doi. org/10.1109/34.55109

16. M.R. Teague, "Image analysis via the general theory of moments", J. Opt. Soc. Am. 70(8), 920 (1980). https://doi.org/10.1364/JOSA.70.000920

17. C. Singh, E. Walia and R. Upneja, "Accurate calculation of Zernike moments", Inform. Sciences 233, 255 (2013). https://doi.org/10.1016/J.INS.2013.01.012

18. A.J. Tencate, J.H. Kalivas and A.J. White, "Fusion strategies for selecting multiple tuning parameters for multivariate calibration and other penalty based processes: a model updating application for pharmaceutical analysis", Anal. Chim. Acta 921, 28 (2016). https://doi.org/10.1016/J.ACA.2016.03.046 


\section{Appendix}

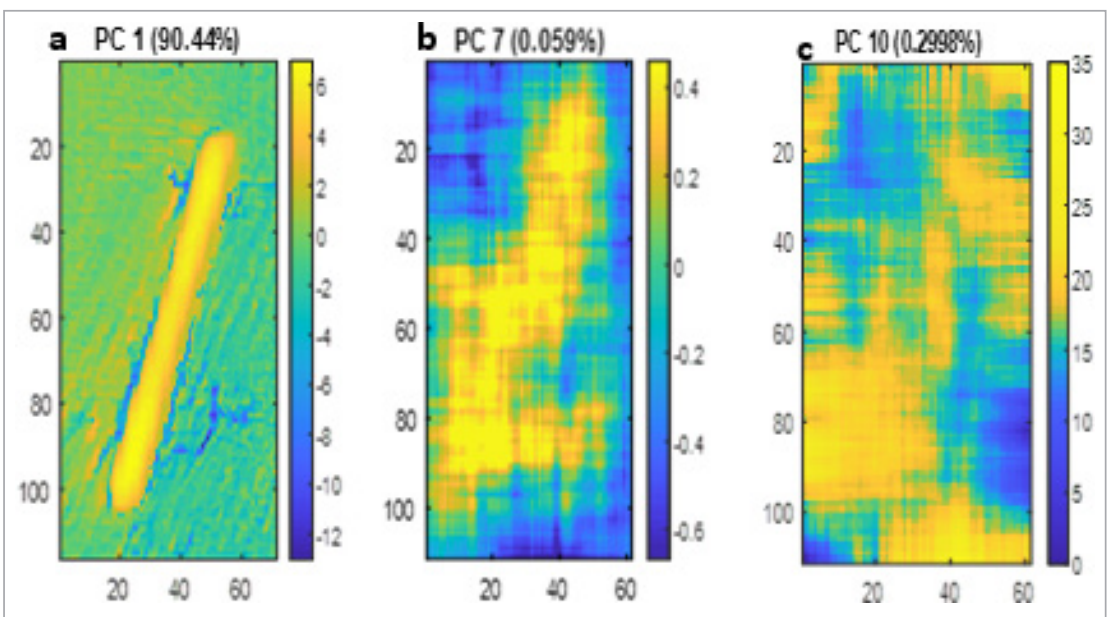

Figure A1. Gun barrel score images of a) clean 1 before defacing, and after defacing for b) phase images, c) Combination of phase and amplitude images.

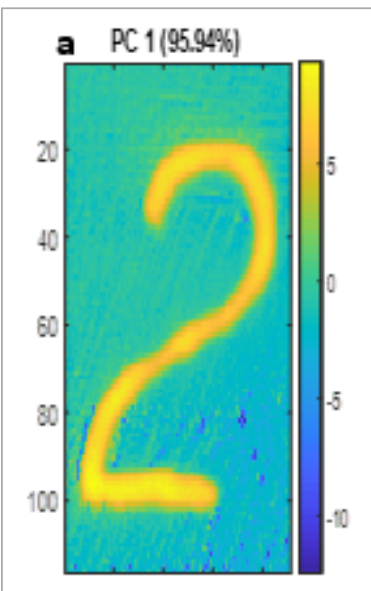

$\begin{array}{llll}20 & 40 \quad 60 & 80\end{array}$

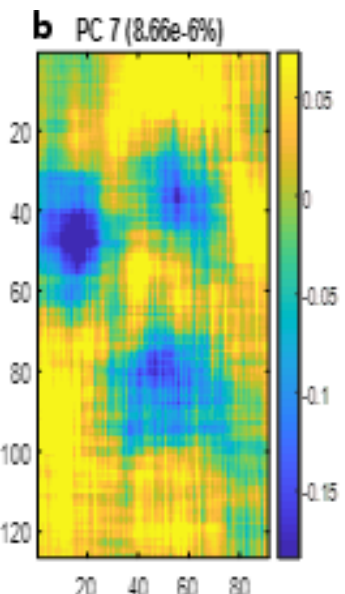

$\begin{array}{llll}20 & 40 \quad 60 \quad 80\end{array}$

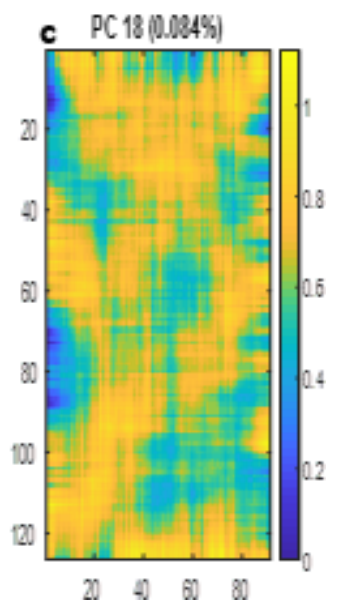

Figure A2. Gun barrel score images of a) clean 2 before defacing, and after defacing for b) phase images, c) combination of phase and amplitude images. 